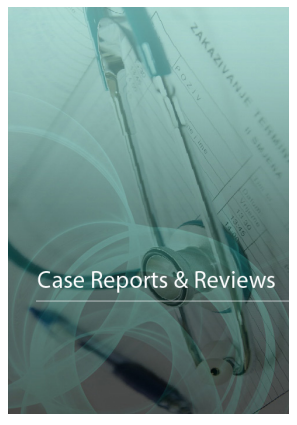

Correspondence

Dr. Chen Bo-Nien

Department of Otolaryngology-Head and Neck Surgery, Hsinchu MacKay Memorial Hospital, No. 690, Sec. 2, Guangfu Rd., East Dist., Hsinchu 30071, Taiwan. Tel: +886 36119595; Fax: +886 36110900

E-mail: chenbonien@yahoo.com.tw

- Received Date: 29 May 2020;

- Accepted Date: 24 June 2020;

- Publication Date: 30 June 2020.

Keywords

Concurrent chemoradiotherapy; natural killer/T-cell lymphoma; postradiation neoplasia; teratocarcinosarcoma.

Copyright

(c) 2020 Science Excel. This is an openaccess article distributed under the terms of the Creative Commons Attribution 4.0 International license.

\section{Sinonasal Teratocarcinosarcoma After Concurrent Chemoradiotherapy for Nasal-Type Natural Killer/ T-Cell Lymphoma: A Rare Case Report}

\author{
Chen Bo-Nien ${ }^{1,2}$ \\ 'Department of Otolaryngology-Head and Neck Surgery, Hsinchu MacKay Memorial Hospital, Hsinchu, Taiwan \\ ${ }^{2}$ Mackay Medical College, New Taipei, Taiwan
}

\begin{abstract}
Teratocarcinosarcoma (TCS) is an extremely rare and aggressive malignant neoplasm with uncertain histogenesis. Overall, fewer than 100 cases of TCS have been reported in English research. This article reports a case of a 19-year-old male patient who was cured of nasal-type natural killer/T-cell (NK/Tcell) lymphoma through concurrent chemoradiotherapy (CCRT). After 13 years, at the age of 32, he developed sinonasal teratocarcinosarcoma (SNTCS) and subsequently underwent endoscopic sinus surgery and CCRT. The patient tolerated the treatment well, and symptoms were resolved. Tumor recurrence was not observed during 12-month posttherapy follow-up endoscopy and magnetic resonance imaging. This is the first reported case of SNTCS development after CCRT for nasal-type NK/T-cell lymphoma. This report highlights the importance of considering the effects of CCRT on SNTCS development and expands the spectrum of reported radiation-induced malignancies in the sinonasal tract.
\end{abstract}

\section{Introduction}

Teratocarcinosarcoma (TCS) was first described as a teratoid carcinosarcoma by Shanmugaratnam et al in 1983, and the term TCS was coined by Heffner and Hyams in 1984 [1]. It is an extremely rare and aggressive malignant neoplasm with uncertain histogenesis [2]. Overall, fewer than 100 cases of TCS have been reported in English research [1-3]. most of which were observed to occur not only in the sinonasal tract but also in the nasopharynx, oral cavity, hypopharynx, ovary, and uterine cervix $[1,3]$. Herein, we report a unique case of a 32-year-old man with sinonasal teratocarcinosarcoma (SNTCS) who had received concurrent chemoradiotherapy (CCRT) for nasal-type natural killer/T-cell (NK/ T-cell) lymphoma 13 years prior.

\section{Case Report}

A 19-year-old Taiwanese man had nasal-type NK/T-cell lymphoma, stage IeA (classified using the Ann Arbor staging system) and was subsequently treated with CCRT. The chemotherapy regimen of CCRT was etoposide, cisplatin, and ifosfamide every 3 weeks (4 courses in total). The type of CCRT radiotherapy used was intensity-modulated radiation therapy (IMRT) administered at a dose of 70 Gy (2 Gy/ fraction; Monday to Friday for 7 weeks) to the primary tumor.

After treatment, neither local recurrence nor distant metastasis was observed at routine follow-ups. At 32 years old, he visited our hospital and reported experiencing blood- tinged discharge from the right nostril for 1 week. Sinoscopic examination revealed bloody mucoid nasal discharge drainage from the right superior meatus (Figure 1). Computed tomography (CT) revealed a $1.2 \times 1.7 \mathrm{~cm}^{2}$ moderately enhanced lesion in the right posterior ethmoid sinus (Figure 2).

Subsequently, the patient underwent endoscopic sinus surgery for lesion resection. Postoperatively, histopathological sections revealed mixed adenosquamous and small-cell neuroendocrine carcinoma with concomitant low-grade fibroblastic-type sarcoma in the stromal part (Figure 3). Histopathological examination and immunohistochemical (IHC) staining results (CK, CK7, p63, p40, synaptophysin, CD56, GFAP, and p16; Figure 4) revealed that the lesion was consistent with a SNTCS.

Moreover, a bone scan, chest and abdomen $\mathrm{CT}$, and positron emission tomography yielded negative results for a metastatic disease. Subsequently, the patient underwent adjuvant CCRT. The chemotherapy regimen of CCRT was cisplatin $\left(75 \mathrm{mg} / \mathrm{m}^{2}\right.$ on Monday) every 3 weeks ( 3 courses in total). The radiotherapy of CCRT involved applying radiation at a dose of $60 \mathrm{~Gy}(2$ Gy/fraction; from Monday to Friday for 6 weeks) to the surgical sinus bed. The patient tolerated the treatment well, and symptoms were resolved.

Tumor recurrence was not observed during posttherapy endoscopy and magnetic resonance imaging at the 12-month follow-up, and the patient resumed a normal life. 


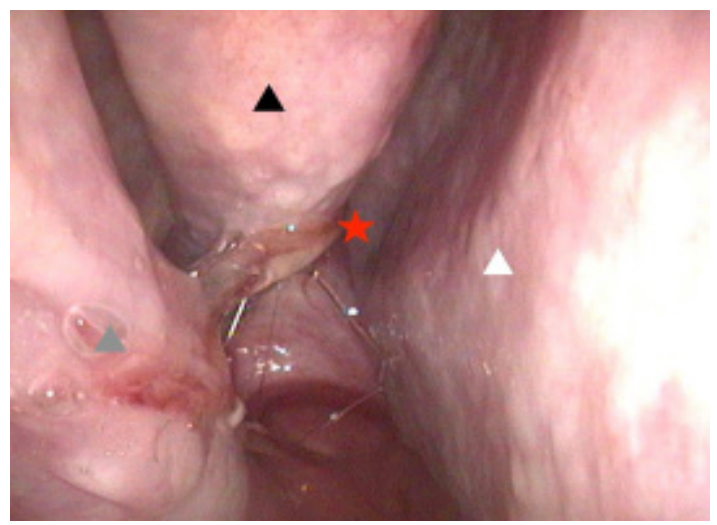

Figure 1. Sinoscopic examination revealed bloody mucoid nasal discharge (red asterisk) drainage from the right superior meatus (black triangle: right middle turbinate; gray triangle: right inferior turbinate; white triangle: nasal septum).

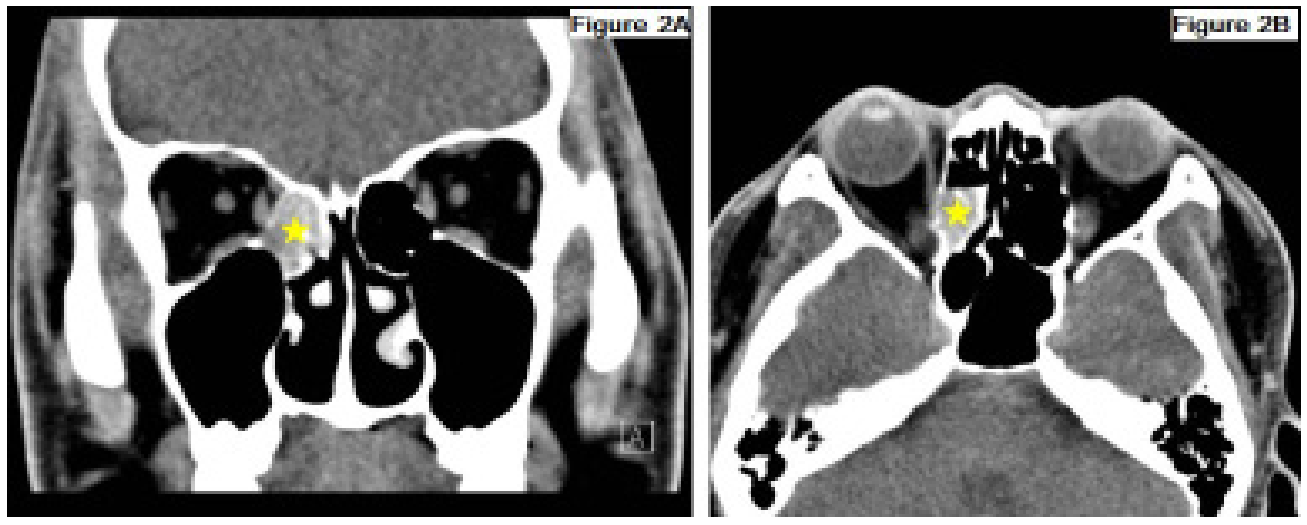

Figure 2. Preoperative (A) coronal and (B) axial CT images revealed a $1.2 \times 1.7 \mathrm{~cm}^{2}$ moderately enhanced lesion (yellow asterisk) in the right posterior ethmoid sinus.
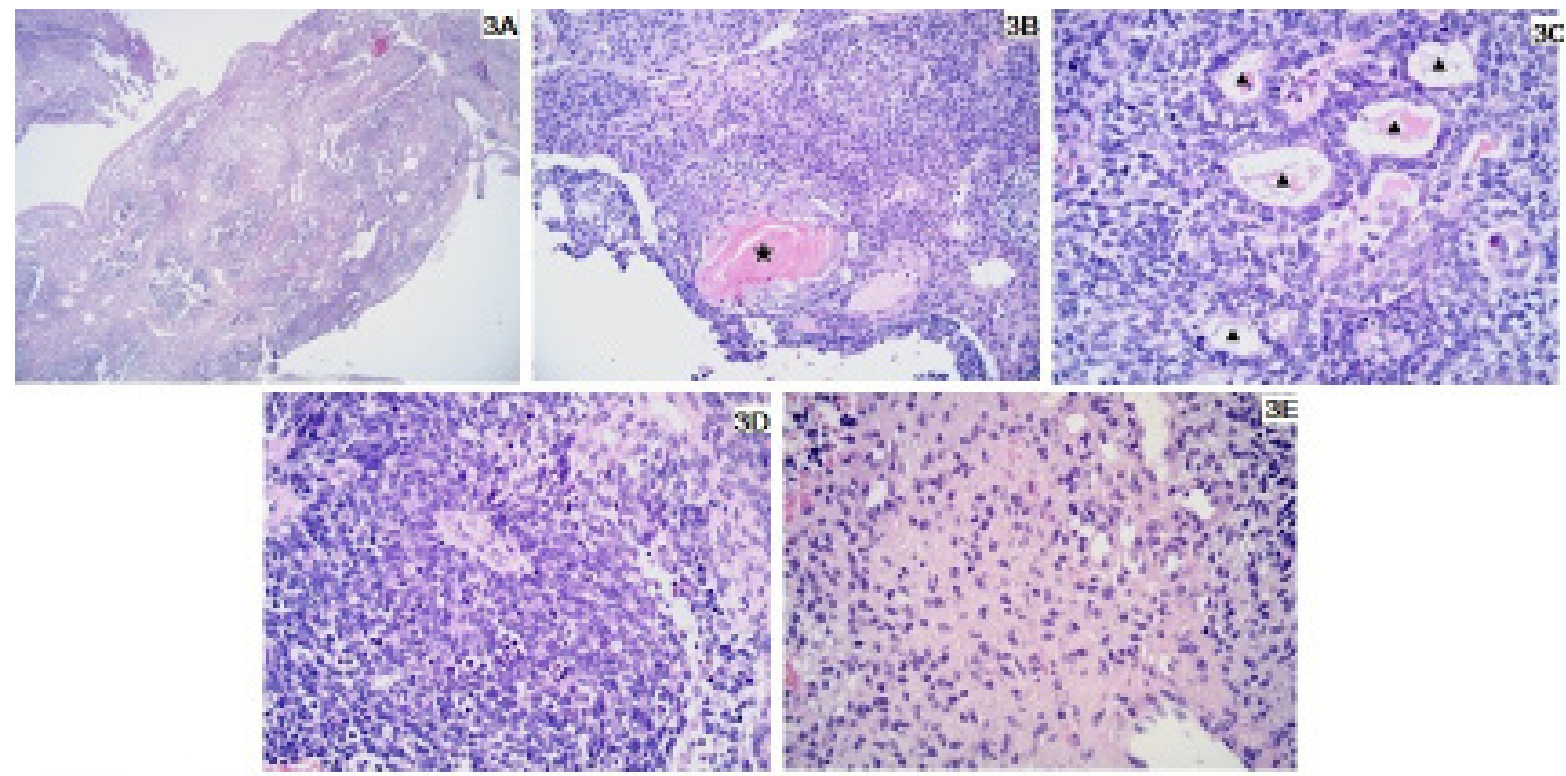

Figure 3. Histopathological sections of the lesion. (A) At a low magnification, the lesion appeared to be a mixed adenosquamous and small-cell neuroendocrine carcinoma with a concomitant low-grade fibroblastic-type sarcoma in the stromal part (hematoxylin and eosin [H\&E], 40× magnification). At a high magnification, the epithelioid cells revealed (B) squamous differentiation (focally with keratinizing features, black asterisk; H\&E, 100× magnification) or (C) partially formed small glandular lumens (black triangles; H\&E, 200× magnification). (D) The small cells had dark nuclei and exhibited frequent apoptosis and occasional necrosis (H\&E, 200× magnification). (E) The intervening/surrounding stroma mostly also exhibited hypo- to occasionally hyper-cellular growth of fairly uniform fibroblast-like cells (probably the primitive to mildly differentiating mesenchymal cells; H\&E, 200× magnification).. 

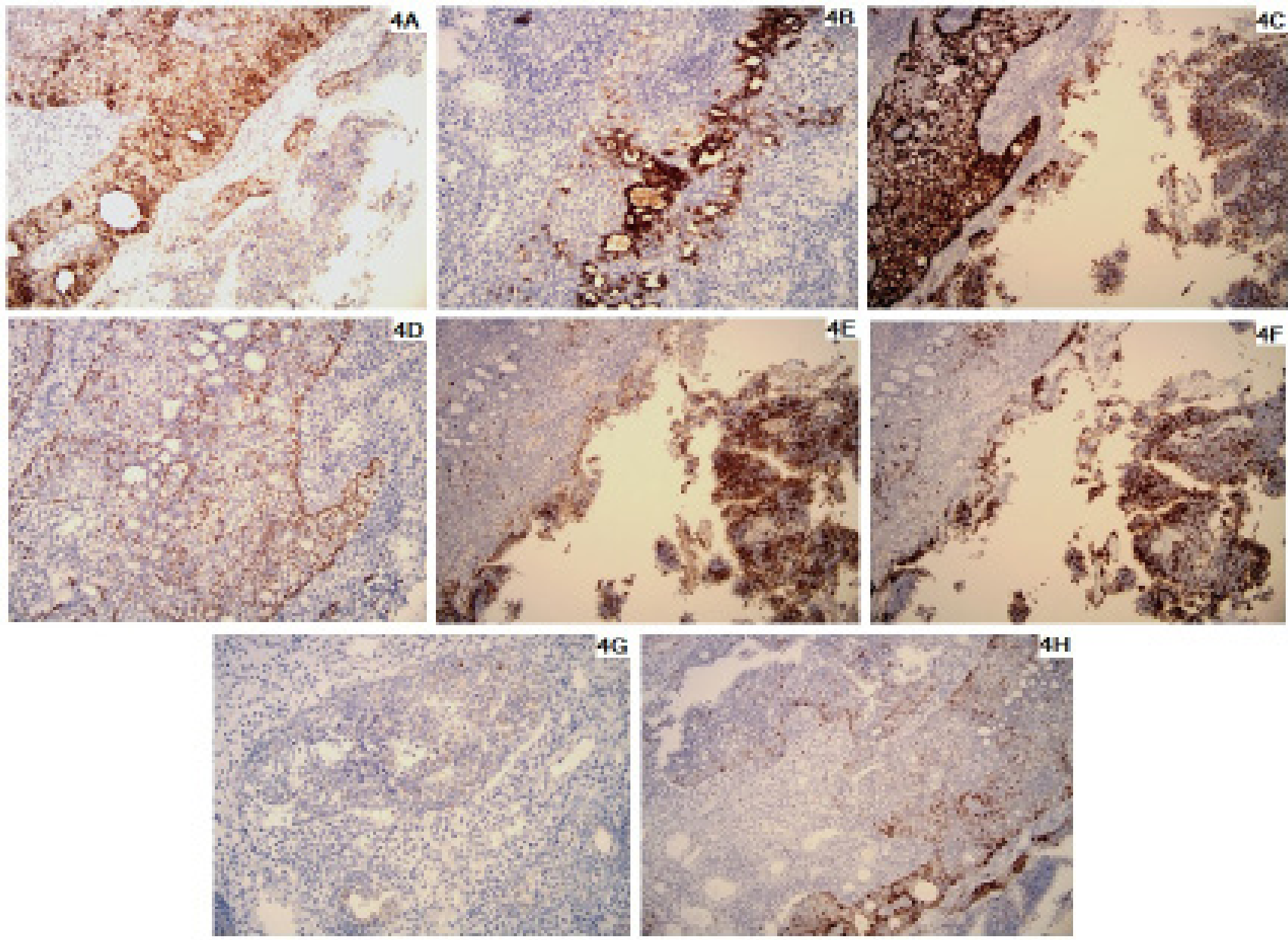

Figure 4. IHC staining of the lesion. (A) Nearly all non-small-cell epithelial tumors test positive for CK, and small-cell epithelial tumors mostly have dot-like staining for CK. (B) CK7 highlights the gland-forming tumor cells. (C) Positive staining for p63 is noted in many non-small-cell epithelial tumors and in some small-cell epithelial tumors. (D) Positive staining for p40 is mainly observed in some non-small-cell epithelial tumors. Small-cell tumors frequently test positive for synaptophysin (E) and CD56 (F).

(G) Some cells of tumor islands are weakly to moderately stained with GFAP. (H) Some tumor cells test positive for p16.

\section{Discussion}

We report a rare case of SNTCS of posterior ethmoid sinus in a 32-year-old man presenting with blood-tinged nasal discharge. SNTCS is almost merely observed in adults aged 54.5 years on average and is mainly noted in men (87\%) [4]. It is most commonly found in the sinonasal tract, with involvement of the ethmoid sinus in approximately half of patients and of the maxillary sinus in approximately one-quarter of patients. Intracranial extension is quite common, given the invasive nature of the tumor [2]. Patients most commonly present with recurrent epistaxis, nasal stuffiness, facial pain, headache, or visual symptoms. Vague symptoms, presence of an air-filled cavity allowing silent growth, and rare pathology make SNTCS diagnosis difficult, and most patients with SNTCS have progressed to the advanced stage by the time of diagnosis [3]. However, in the present case, a comprehensive sinoscopy helps us detect the lesion early.

Little is known regarding the genetic factors regulating SNTCS so far. Birkeland et al identified a p.S45F activation mutation in $\beta$-catenin in SNTCS that resulted in $\beta$-catenin overexpression. These findings indicate a role of the $\mathrm{Wnt} / \beta$-catenin pathway in SNTCS tumorigenesis [5]. Histogenetically, this tumor is unlikely to be of germ cell origin but probably originates from a primitive cell in the olfactory membrane that not only replicates the neuroectodermal features of olfactory neuroblastoma but also can differentiate into divergent somatic cell types [6].
Radiotherapy could potentially lead to unrecognized mutations, predisposing a patient to a second malignancy. In the present case, SNTCS was suspected as a second sinonasal malignancy induced by radiotherapy previously used to treat the patient's nasal-type NK/Tcell lymphoma. Thus, this represents a rare late-onset complication of radiotherapy. Although CCRT is currently the gold standard of treatment for nasal-type NK/T-cell lymphoma, such "cured" patients are at risk of a second malignancy. The risk of radiotherapy should be explicitly communicated to patients and their families before initiation. This report highlights the importance of considering the effect of CCRT on SNTCS occurrence and expands the spectrum of reported radiation-induced malignancies in the sinonasal tract.

Histologically, SNTCS consists of epithelial components and 2 or more mesenchymal components, such as fibroblasts, cartilage, muscular and bony tissues [1]. The epithelial component ranges from benign glandular epithelium to malignant olfactory epithelium. The mesenchymal element is composed of benign or malignant spindleshaped cells in a myxoid matrix. Nests of small blue malignant cells may coexist and comprise the neuroepithelial element [3]. Because SNTCS exhibits histologically diverse features, such as mature/ benign and immature/malignant epithelial and mesenchymal components, pathologists must distinguish SNTCS from carcinosarcoma, teratoma, teratoma with malignant transformation, and peripheral primitive neuroectodermal tumors [1]. Due to the large number of cell types in SNTCS, biopsy alone is not enough. 
SNTCS requires sufficient sampling for proper diagnosis $[2,7]$.

For the diagnosis of SNTCS, IHC staining is necessary. IHC staining is dependent on the cell type; epithelial components are positive for cytokeratin and epithelial membrane antigens; neuroepithelial components are positive for neuron-specific enolase, CD99, chromogranin, synaptophysin, glial fibrillary acidic protein, and S-100 protein; mesenchymal components are positive for vimentin and depending on the cell type, may be reactive to myogenic markers or smooth muscle actin [6].

In the absence of guidelines, no consensus has been reached regarding the treatment algorithm of SNTCS. However, the most common treatment is complete resection followed by radiotherapy [3]. IMRT can be used, given its excellent outcomes with minimal side effects [2]. The use of elective nodal radiotherapy may be justified in SNTCS, which has a high probability of locoregional failure [3]. The role of systemic therapy remains unclear. However, few studies have supported adjuvant chemotherapy $[1,4]$. Platinum is one of the most common drugs used in most series, in concurrence with either radiation or another chemotherapy drug [3]. In the present case, the patient underwent endoscopic sinus surgery for lesion resection and adjuvant CCRT. Tumor recurrence was not observed during posttherapy endoscopy and magnetic resonance imaging at the 12-month follow-up. However, long-term follow-up is required to assess for late recurrence.

Most patients with SNTCS develop symptoms at an advanced stage and have an infiltrative spread pattern, resulting in poor prognosis (average survival of 1.7 years) due to frequent recurrence and metastasis [1,2]. With a mean follow-up of 38.9 months, Misra et al reported that $24.4 \%, 9.5 \%$, and $6.9 \%$ of patients had local recurrence, metastatic disease, and both, respectively. The lungs are the most common site of distant metastasis [4]. Active follow-up is necessary to detect early recurrence. Early diagnosis and management before regional spread can improve prognosis [6].

\section{Conclusion}

This is the first reported case of SNTCS development after CCRT for nasal-type NK/T-cell lymphoma. This report highlights the importance of considering the effect of CCRT on SNTCS occurrence and expands the spectrum of reported radiation-induced malignancies in the sinonasal tract.

\section{References}

1. Ito K, Yano M, Ogasawara A, Miwa M, Kozawa E, Yasuda M. A unique uterine cervical "teratocarcinosarcoma": a case report. Diagn Pathol. 2019;14(1):122.

2. Foong YC, Murdolo V, Naiman N, Hepner L, Awad R. Sinonasal teratocarcinosarcoma: a case report. J Med Case Rep. 2017;11(1):167.

3. Tandon S, Gairola M, Ahlawat P, Sharma K, Barik S, Sachdeva N, et al. Sinonasal teratocarcinosarcoma treated with surgery followed by volumetric modulated radiotherapy: a case report with review of literature. Radiat Oncol J. 2018;36(4):341-7.

4. Misra P, Husain Q, Svider PF, Sanghvi S, Liu JK, Eloy JA. Management of sinonasal teratocarcinosarcoma: a systematic review. Am J Otolaryngol. 2014;35(1):5-11.

5. Birkeland AC, Burgin SJ, Yanik M, Scott MV, Bradford CR, McHugh JB, et al. Pathogenetic Analysis of Sinonasal Teratocarcinosarcomas Reveal Actionable beta-catenin Overexpression and a beta-catenin Mutation. J Neurol Surg B Skull Base. 2017;78(4):346-52.

6. Chakraborty S, Chowdhury AR, Bandyopadhyay G. Sinonasal teratocarcinosarcoma: Case report of an unusual neoplasm. J Oral Maxillofac Pathol. 2016;20(1):147-50.

7. Elia CJ, Cabanne M, Piao Z, Lee A, Goldenberg T, Chhabra V. A rare case of intracranial teratocarcinosarcoma: Case report and review of literature. Surg Neurol Int. 2018;9:167. 